\title{
ECOEFICIÊNCIA DAS PEQUENAS PROPRIEDADES SUINÍCOLAS COOPERATIVISTAS CATARINENSES: INFLUÊNCIA DA CERTIFICAÇÃO PELO PROGRAMA PROPRIEDADE RURAL SUSTENTÁVEL
}

\author{
ECOEFFICIENCY OF CATARINIAN COOPERATIVE SWINE FARMS: \\ INFLUENCE OF CERTIFICATION BY THE SUSTAINABLE RURAL PROPERTY \\ PROGRAM
}

\section{ECOEFICIENCIA DE LAS PEQUEÑAS PROPIEDADES PORCINAS COOPERATIVAS CATARINAS: INFLUENCIA DE LA CERTIFICACIÓN DEL PROGRAMA DE PROPIEDAD RURAL SOSTENIBLE}

\author{
Kátia Dalcero ${ }^{1}$ \\ ORCID: https://orcid.org/0000-0002-2123-3343 \\ Denize Demarche Minatti Ferreira ${ }^{2}$ \\ ORCID: https://orcid.org/0000-0002-4661-9672
}

Submissão: 12/11/2020 / Aceito: 11/05/2021 / Publicado: 04/02/2021.

\begin{abstract}
Resumo
O aumento da produção de carne suína ocorrido nos últimos anos no país tem demandado maior uso de recursos naturais e, consequentemente, a necessidade de implementação de práticas e programas de manejo sustentável. Nesse contexto, o presente estudo verificou a influência da certificação pelo Programa Propriedade Rural Sustentável nos índices de ecoeficiência das pequenas propriedades suinícolas cooperativistas catarinenses. A amostra do estudo é formada pelas propriedades vinculadas a Cooperativa A1, localizada na região Oeste do estado de Santa Catarina. A coleta de dados foi realizada por meio de visitas nas propriedades selecionadas e dos relatórios de fechamento de lote por propriedade rural fornecidos pela agroindústria entre 2015 e 2018. O cálculo dos índices de ecoeficiência foi realizado por meio da Análise Envoltória de Dados (DEA) e para avaliar a influência da certificação nos índices de ecoeficiência foi utilizado o modelo de regressão múltipla com dados em painel (pooled). Os resultados apontaram que as propriedades apresentam níveis altos de ecoeficiência e que as propriedades rurais certificadas obtiveram índices maiores de ecoeficiência em relação àquelas não certificadas. Esses resultados indicam que os incentivos por intermédio de certificação de cooperativas se mostram promissores tanto para as pequenas propriedades quanto para o meio ambiente.
\end{abstract}

Palavras-chaves: Ecoeficiência; Propriedades Suinícolas Cooperativistas; DEA.

\begin{abstract}
The increase in pork production in the country in recent years has demanded a greater use of natural resources and, consequently, the need to implement sustainable management practices and programs. In this context, the present study verified the influence of certification by the Sustainable Rural Property Program influences the eco-efficiency

\footnotetext{
${ }^{1}$ Mestre em Contabilidade. Doutoranda no Programa de Pós-Graduação em Contabilidade da Universidade Federal de Santa Catarina. E-mail: katiadalcero13@gmail.com

${ }^{2}$ Doutora em Engenharia e Gestão do Conhecimento. Professora do Departamento de Ciências Contábeis da Universidade Federal de Santa Catarina. E-mail: denize.minatti@ufsc.br
} 
indexes of small cooperative swine farms in Santa Catarina. The study sample is composed of properties linked to the Cooperativa A1, in western Santa Catarina state. Data collection was performed through visits to the selected properties and the reports of lot closure by rural property provided by the agro-industry, between 2015 and 2018. The eco-efficiency indexes were estimated through Data Envelopment Analysis (DEA) and to assess the influence of certification on the eco-efficiency indexes, a multiple regression model with panel data (pooled) was used. Results showed that the properties present high levels of ecoefficiency and that certified rural properties present higher rates of eco-efficiency compared to those not certified. These results may indicate that incentives through cooperative certification can be promising for both small properties and the environment.

Keywords: Eco-efficiency; Agricultural Cooperatives; DEA.

\section{Resumen}

El aumento de la producción porcina que se ha dado en el país en los últimos años ha exigido un mayor uso de los recursos naturales y, en consecuencia, la necesidad de implementar prácticas y programas de manejo sustentable. En este contexto, este estudio verificó la influencia de la certificación del Programa de Propiedad Rural Sostenible en los índices de ecoeficiencia de las pequeñas explotaciones porcinas cooperativas de Santa Catarina. La muestra de estudio está formada por predios vinculados a la Cooperativa A1, ubicada en la región occidental del estado de Santa Catarina. La recolección de datos se realizó a través de visitas a predios seleccionados e informes de cierre de lotes por predio rural proporcionados por la agroindustria entre 2015 y 2018. El cálculo de índices de ecoeficiencia se realizó mediante Análisis Envolvente de Datos (DEA) y para evaluar la influencia de la certificación en índices de ecoeficiencia, se utilizó un modelo de regresión múltiple con datos de panel (agrupados). Los resultados mostraron que las propiedades presentan altos niveles de ecoeficiencia y que las propiedades rurales certificadas tuvieron mayores índices de ecoeficiencia en comparación con las que no fueron certificadas. Estos resultados indican que los incentivos a través de la certificación de cooperativas son prometedores tanto para las pequeñas propiedades como para el medio ambiente.

Palabras chave: Ecoeficiencia; Propiedades cooperativas de cerdos; DEA.

\section{INTRODUÇÃO}

O agronegócio tem fundamental importância para a economia brasileira beneficiando diferentes grupos da sociedade (COHEN, 2019). De acordo com o Centro de Estudos Avançados em Economia Aplicada (CEPEA), no ano de 2019 o agronegócio teve uma participação de R 1.552.995 bilhões no Produto Interno Bruto (PIB), apresentando um aumento de 3,81\% em relação ao ano anterior. Já a pecuária corresponde ao montante de $\mathrm{R} \$ 494.756$ bilhões, incluindo todas as atividades envolvidas (insumos, agropecuária, indústria e serviços), o que representa um crescimento de 23,71\% em relação à 2018.

No que se refere à carne suína, foram abatidos 11,87 milhões de animais no quarto trimestre de 2019, demonstrando um aumento de 6,1\% em relação ao mesmo período de 2018 (IBGE, 2020). No que se refere à distribuição geográfica, a região Sul é responsável 
por $65,3 \%$ dos abates, sendo Santa Catarina o Estado líder com o percentual de 26,8\% (IBGE, 2019). Lopes (2019, p. 2) destaca que "pesaram para esse aumento os reflexos positivos para a demanda e preços da epidemia de peste suína na China e sobre o mercado global de carnes". O aumento da produção de carne suína influencia diretamente a demanda pelos recursos naturais (LASSALETTA et al., 2019), onde os mercados aprimoram os critérios de utilização dos mesmos. Assim, surge a necessidade de implementar práticas e programas de manejo sustentável que incentivem os produtores à inovação que proporcione benefícios econômicos, ambientais e sociais, viabilizando a continuidade da atividade e o desenvolvimento sustentável (LUBELL; HILLIS; HOFFMAN, 2011).

Um dos instrumentos que afere a relação entre os três aspectos da sustentabilidade (econômicos, ambientais e sociais) é a ecoeficiência. Esta ferramenta avalia a sustentabilidade e o uso eficiente dos recursos naturais nas atividades produtivas em diferentes setores da economia (HUPPES; ISHIKAWA, 2005), bem como auxilia as organizações na busca do desenvolvimento sustentável, na criação de valor de suas atividades e a avaliar o impacto possível em funcionários, clientes, fornecedores, meio ambiente e comunidade (ISAR, 2004).

As organizações agropecuárias utilizam a avaliação da ecoeficiência para estimar processos produtivos, implantar novas tecnologias e desenvolver políticas que visem o crescimento sustentável (VAN PASSEL et al., 2007). Neste contexto, os programas de certificação trazem benefícios financeiros, econômicos e melhorias ambientais para as propriedades agropecuárias (TRAN; GOTO, 2018).

A avaliação da ecoeficiência permite melhores condições no processo de tomada de decisão, introdução de novas políticas e investimentos e, inovações tecnológicas na busca da criação de valor sustentável (SCHALTEGGER; BURRITT, 2010). Dessa forma, são necessárias medidas que verifiquem a contribuição das organizações para a sustentabilidade e nessa linha, van Passel et al. (2007) afirmam que as organizações contribuem para a sustentabilidade quando os benefícios gerados (econômicos, sociais e ambientais) excedem a soma dos custos da atividade. Logo, a implantação de mecanismos de avaliação da sustentabilidade nas organizações agropecuárias, em especial nas propriedades rurais, é incentivada pelas cooperativas, uma vez que são responsáveis pela inserção dos pequenos e médios produtores nos mercados. Deste modo, as cooperativas agropecuárias são 
importantes para o desenvolvimento econômico, social e ambiental no país (HUETH; REYNOLDS, 2011; RICHTER LONDERO et al., 2019).

O Programa Propriedade Rural Sustentável - desenvolvido no ano de 2016 por uma agroindústria da região Oeste de Santa Catarina - é um exemplo de implementação de mecanismos de avaliação da sustentabilidade e busca a implantação do manejo produtivo sustentável de forma integrada com as cooperativas filiadas e os produtores rurais cooperativistas. Neste, as propriedades certificadas recebem incentivos financeiros pela aplicação de boas práticas de manejo na propriedade de acordo com o manual por eles desenvolvido. O certificado foi conferido a 16 propriedades no ano de 2016, a 53 propriedades em 2017 e, no ano de 2018, a 128 propriedades (BUSINESS, 2019).

A análise da ecoeficiência do processo produtivo da criação de suínos, sobretudo na região Oeste catarinense, é importante para auxiliar no desenvolvimento de políticas que contribuam para o crescimento econômico e a preservação dos recursos naturais. Diante do exposto, a pergunta que norteia a presente pesquisa é: Qual a influência da certificação pelo Programa Propriedade Rural Sustentável nos índices de ecoeficiência das pequenas propriedades suinícolas cooperativistas catarinenses? Logo, o objetivo deste estudo é analisar a influência da certificação pelo Programa Propriedade Rural Sustentável nos índices de ecoeficiência das pequenas propriedades suinícolas cooperativistas catarinenses.

O estudo se justifica pela importância de avaliar a sustentabilidade das propriedades suinícolas através da ecoeficiência, o que possibilita o desenvolvimento e aplicação de novos e mais adequados processos produtivos que geram benefícios econômicos, ambientais e sociais. Ainda, visa apoiar a formulação de políticas que reduzam as pressões ambientais restritivas à atividade econômica (PICAZO-TADEO; GÓMEZ-LIMÓN; REIGMARTÍNEZ, 2011), pois não há uma base para avaliar os procedimentos das três dimensões da sustentabilidade de modo integrado no contexto das cooperativas agropecuárias (MARCIS; PINHEIRO DE LIMA; GOUVÊA DA COSTA, 2019). Além disso, busca auxiliar na formulação de políticas de incentivos, ao demonstrar os efeitos da certificação para melhoria da sustentabilidade desenvolvida nos níveis microeconômicos.

Ademais, o estudo contribui com as dimensões acadêmica, empresarial e social sobre a ecoeficiência como um instrumento de avaliação da sustentabilidade no setor do agronegócio, principalmente na produção de suínos, uma vez que são poucos aqueles direcionados aos sistemas produtivos (VAN PASSEL et al., 2007; VELEVA; 
ELLENBECKER, 2001) que estimam a alocação dos estoques de capitais na referida atividade (FIGGE; HAHN, 2004; HUPPES; ISHIKAWA, 2005). Logo, busca colaborar com a sociedade, ao discutir e implantar práticas que melhorem a preservação dos recursos naturais, por meio de seu uso eficiente, e ao mesmo tempo gerem benefícios econômicos de curto, médio e longo prazo. Por fim, a implementação dessas práticas e sua avaliação colaboram para a garantia e aplicação do conceito de desenvolvimento sustentável, bem como para a preservação dos recursos naturais essenciais para a vida humana.

\section{FUNDAMENTAÇÃO TEÓRICA}

\section{Medidas de avaliação da sustentabilidade}

Conforme a Teoria dos Capitais, a sustentabilidade pode ser conceitualmente desmembrada em "fraca e forte" de acordo com a substituição dos capitais, que se caracteriza, segundo Norton e Toman (1997) e Stern (1997), como a principal diferença entre as duas. A sustentabilidade "fraca" aborda todos os capitais e a falta de um deles leva a substituição por qualquer outro excedente. A sustentabilidade "forte" determina que os capitais não são substituíveis, assim se tem a necessidade da preservação dos mesmos, onde se complementam entre si (FIGGE; HAHN, 2004).

A avaliação da sustentabilidade pode ser realizada de duas formas: (i) por meio da análise dos impactos ambientais e (ii) ou da orientação pelo ônus ou pelo valor. A primeira analisa os custos e/ou danos causados pela atividade econômica em detrimento a um outro conjunto de impacto ambiental, por considerar que os recursos dos capitais podem ser substituídos uns pelos outros. A segunda analisa "quanto de valor" é criado por um conjunto de impactos ambientais negativos, abordagem que adota a análise da utilização dos recursos em comparação a outras organizações, os quais precisam ser alocados de maneira eficiente (FIGGE; HAHN, 2004).

Estas medidas combinam indicadores das três dimensões da sustentabilidade, e ainda consideram níveis macro e microeconômicos (JOLLANDS; LERMIT; PATTERSON, 2004). Nos últimos anos foram propostas medidas para avaliação da sustentabilidade concentradas nos níveis macroeconômicos, como por exemplo: pegada ecológica, economia genuína, índice de bem-estar econômico sustentável e painel de sustentabilidade (VAN PASSEL et al., 2007). Portanto, é necessário avançar na avaliação em níveis organizacionais e verificar qual é a contribuição das empresas para a 
sustentabilidade, já que as atividades produtivas que mais consomem recursos ocorrem nestas organizações

Van Passel et al. (2007) afirmam que é importante aferir a sustentabilidade das propriedades agropecuárias para fornecer orientação para a tomada de decisão, o que é fundamental para a garantia da continuidade da atividade (DANTSIS et al., 2010). De acordo com Lewandowski, Härdtlein e Kaltschmitt (1999), a sustentabilidade agropecuária é a forma de manejo e utilização do recursos naturais e manutenção dos ecossistemas, com integração econômica, ambiental e social nos níveis locais, nacionais e global sem prejuízos.

A avaliação da sustentabilidade agropecuária é um processo complexo com variáveis inter-relacionadas para auxiliar a tomada de decisão e aprimorar as políticas que incentivem práticas de manejos produtivos menos agressivas ao meio ambiente (Dantsis et al., 2010; Galdeano-Gómez et al., 2017; Sala et al., 2015). O processo leva em consideração as análises dos sistemas produtivos agropecuários (unidade de produção e localização) para avaliação da sustentabilidade (GÓMEZ-LIMÓN; SANCHEZFERNANDEZ, 2010).

\section{Ecoeficiência: Estudos relacionados}

A ecoeficiência é uma das métricas mais utilizadas nas pesquisas acadêmicas para análise da sustentabilidade em distintos setores da economia (FIGGE; HAHN, 2013; HUANG et al., 2018), considerada uma medida relativa de sustentabilidade (FIGGE; HAHN, 2004). O conceito começou a ser abordado na década de 1970 por Freeman, Haveman e Kneese (1973) para verificar a eficiência ambiental dos negócios (MU et al., 2018) pela escassez dos recursos naturais e sua utilização eficiente (FIGGE; HAHN, 2013).

Schaltegger e Sturn (1990) e Schaltegger (1996) voltaram a discutir esta abordagem e agregaram a relação entre desempenho econômico e impactos ambientais negativos das atividades. O conceito se popularizou pelo Conselho Empresarial Mundial para o Desenvolvimento Sustentável para criar um filosofia de gestão para incentivar as organizações na busca integrada de benefícios econômicos com melhorias socioambientais (BONFIGLIO; ARZENI; BODINI, 2017).

A literatura define ecoeficiência como razão do valor econômico agregado por impacto ambiental gerado (YOU; ZHANG, 2016) e a medida é referenciada nos estudos por duas linhas de aplicação, a primeira é a "máxima ecoeficiência” que determina a 
diminuição dos impactos ambientais negativos (DESIMONE; POPOFF, 1997) e a segunda evidencia a proporção do benefício econômico (criação de valor) por impacto ambiental adicionado (FIGGE; HAHN, 2013; PICAZO-TADEO; GÓMEZ-LIMÓN; REIGMARTÍNEZ, 2011; SCHALTEGGER; STURM, 1990).

Figge e Hahn (2004a) destacam três fragilidades na medida de ecoeficiência em relação à contribuição das organizações para a sustentabilidade: (i) é uma medida relativa, não fornecendo informações sobre a eficácia, (ii) os avanços do progresso da ecoeficiência de uma organização, em relação a diminuição de consumo de recursos, podem ser "absorvidos" por outra organização menos ecoeficiente e, (iii) a medida não considera todos os impactos ambientais e sociais simultaneamente.

Picazo-Tadeo et al. (2011) analisaram a ecoeficiência agrícola na produção de cereais em propriedades espanholas por meio da Análise Envoltória de Dados (DEA). Os resultados apontaram que as propriedades rurais são ecoineficientes, principalmente no superávit de nitrogênio e, apresentam pouca diferença entre as pressões ambientais analisadas. Os autores destacaram três razões principais para essa ineficiência: (i) práticas ineficientes de insumos, (ii) falta de conscientização das externalidades ambientais pelos agricultores e, (iii) estrutura não adequada de critérios para tomada de decisão.

You e Zhang (2016) utilizaram DEA para medir a ecoeficiência da produção agrícola intensiva de regiões chinesas e desenvolveram os seguintes critérios: total, técnica e por escala de identificação. Os resultados indicaram que no critério da eficiência total, seis províncias de um total de 31 obtiveram nível máximo e que a eficiência de escala possui índices inferiores aos da eficiência técnica.

Bonfiglio et al. (2017) avaliaram os índices de ecoeficiência de propriedades italianas de 2011 a 2014 e evidenciaram que a maioria das propriedades demonstra índices baixos de ecoeficiência e que, além disso, esta tende a ser maior se os gestores são jovens e participam de cooperativas.

Baum e Bienkowski (2020) avaliaram a ecoeficiência das culturas de milho significativas para exportação da agricultura familiar. Foram selecionadas 69 propriedades rurais localizadas na Polônia utilizando avaliação e custo do ciclo de vida. Os resultados demonstraram que a pegada de carbono foi maior nas propriedades rurais de criação de animais e que a suinocultura apresentou um maior custo. 
A avaliação dos índices de ecoeficiência e seus determinantes na atividade agropecuária é justificada pela relação direta da atividade com o uso dos recursos naturais que são limitados para o fornecimento de bens básicos para atender a demanda da sociedade (GODOY-DURÁN et al., 2017). Os autores verificaram que a adoção de programas de certificação e a associação a cooperativas possuem influência positiva na melhoria dos índices de ecoeficiência.

Grzelak, Guth, Matuszczak e Brelik (2019) compararam dois sistemas de produção levando em consideração o tamanho da propriedade, capital e mão-de-obra, partindo do pressuposto que as propriedades intensivas em mão-de-obra possuíam melhores índices de sustentabilidade do que as propriedades maiores e mais intensivas em capital. Porém, os resultados se contrapuseram aos pressupostos, uma vez que as maiores propriedades obtiveram resultados mais significantes de ecoeficiência. Assim, com base nos argumentos dos autores, apresenta-se a seguinte hipótese de pesquisa:

H1: Propriedades certificadas apresentam índices maiores de ecoeficiência em relação às propriedades não certificadas.

\section{PROCEDIMENTOS METODOLOGICOS}

\section{Amostra e coleta de dados}

A população é formada pelas pequenas propriedades suinícolas integradas à cooperativa agropecuária CooperA1 com matriz em Palmitos (SC) com aproximadamente 8.686 cooperados e faturamento de $\mathrm{R} \$ 1.330 .082 .993 \mathrm{em} 2019$.

A CooperA1 participa do Programa Propriedade Rural Sustentável desenvolvido pela AURORA que incentiva as práticas de produção sustentável em todas as atividades (leite, suínos, aves e grãos) desenvolvidas pelas propriedades filiadas às cooperativas. Para obter a certificação do Programa Propriedade Rural Sustentável, as propriedades são auditadas pela AURORA, por meio da aplicação de um checklist que verifica as práticas de manejo, planejamento estratégico e os indicadores econômico-financeiros e produtividade, além das demonstrações contábeis. A propriedade é certificada quando todas as atividades obtêm pontuação superior a 85 pontos de uma escala de 1 a 100.

O presente estudo analisou a ecoeficiência em propriedades certificadas e nãocertificadas localizadas nos municípios de Caibi, Palmitos e Riqueza e se sustenta em Galdeano-Gómez et al. (2017) que afirmam que para verificar a sustentabilidade 
agropecuária, as propriedades devem estar localizadas em regiões similares para que seja possível a comparação (Tabela 1).

As propriedades localizadas em Caibi são: PROP J; PROP K; PROP P e PROP R, em Palmitos: PROP B; PROP C; PROP F; PROP G; PROP I; PROP N; PROP Q; PROP U e PPROP Y e, as situadas em Riqueza: PROP A; PROP D; PROP H; PROP L; PROP M; PROP O; PROP T; PROP V; PROP W e PROP X.

Para a seleção das propriedades que compõem a amostra foram observados os critérios: (i) pertencentes a mesma região ou município e (ii) tamanho (hectares).

Tabela 1. Amostra da pesquisa

\begin{tabular}{ccc}
\hline Filiais & Propriedades Certificadas & Propriedades Não-certificadas \\
\hline Caibi & 2 & 2 \\
Palmitos & 5 & 4 \\
Riqueza & 5 & 5 \\
Total & 12 & 11 \\
\hline
\end{tabular}

Fonte: Dados da Pesquisa (2020)

A coleta de dados foi realizada por meio de visitas nas propriedades selecionadas e as variáveis de fontes secundárias foram obtidas dos relatórios de fechamento de lote por propriedade fornecidos pela agroindústria do período compreendido entre 2015 e 2018.

Inicialmente, foram definidos os indicadores (das dimensões ambientais, sociais e econômicas) da literatura para os cálculos das pressões ambientais, valor econômico agregado e análises adicionais sobre as variáveis de ecoeficiência (Tabela 2). Os estudos que sustentam a avaliação com base em ecoeficiência são de Mu et al. (2018), PicazoTadeo et al. (2011) e You e Zhang (2016) que avaliaram os índices de ecoeficiência em culturas agrícolas, por meio da utilização do método DEA.

As informações econômico-financeiras utilizadas são: (i) preço recebido por carcaça; (ii) vendas; e (iii) subsídios, e como indicadores socioambientais: (i) idade do produtor; (ii) emissão de gases de efeito estufa; e (iii) consumo hídrico. 
Tabela 2. Variáveis do estudo

\begin{tabular}{|c|c|c|c|}
\hline Indicadores & Descrição & Estudos & Variável \\
\hline & $R E C V$ & Grzelak et al. (2019) & \\
\hline ECO & $\overline{E G E E+C H+C A M}$ & $\begin{array}{c}\text { Baum e Bieńkowski } \\
\text { (2020) }\end{array}$ & Dependente \\
\hline EGEE & $\begin{array}{l}\text { Quantidade de dejetos } \\
\text { produzidos }\end{array}$ & $\begin{array}{l}\text { Baum e Bieńkowski } \\
(2020)\end{array}$ & $\begin{array}{c}\text { Utilizada para o cálculo da } \\
\text { variável dependente (índice } \\
\text { de Ecoeficiência) }\end{array}$ \\
\hline $\mathbf{C H}$ & $\begin{array}{c}\text { Quantidade de água } \\
\text { utilizada e/ou valor total } \\
\text { pago }\end{array}$ & $\begin{array}{l}\text { Bonfiglio et al. (2017); } \\
\text { ISAR (2004); } \\
\text { Kuosmanen e } \\
\text { Kortelainen (2005); Mu } \\
\text { et al. (2018); Picazo- } \\
\text { Tadeo et al. (2011) }\end{array}$ & $\begin{array}{c}\text { Utilizada para o cálculo da } \\
\text { variável dependente (índice } \\
\text { de Ecoeficiência) }\end{array}$ \\
\hline QSA & $\begin{array}{l}\text { Capacidade de } \\
\text { alojamento da pocilga }\end{array}$ & $\begin{array}{l}\text { Manual Propriedade } \\
\text { Sustentável (2019) }\end{array}$ & $\begin{array}{c}\text { Utilizada para o cálculo da } \\
\text { variável dependente (índice } \\
\text { de Ecoeficiência) }\end{array}$ \\
\hline QSV & $\begin{array}{c}\text { Quantidade de suínos } \\
\text { entregues a } \\
\text { agroindústria }\end{array}$ & $\begin{array}{l}\text { Manual Propriedade } \\
\text { Sustentável (2019) }\end{array}$ & $\begin{array}{l}\text { Utilizada para o cálculo da } \\
\text { variável dependente (índice } \\
\text { de Ecoeficiência) }\end{array}$ \\
\hline RECV & $\begin{array}{l}\text { Receita total da venda } \\
\text { de suínos, considera os } \\
\text { valores recebidos de } \\
\text { subsídios }\end{array}$ & $\begin{array}{l}\text { Bonfiglio et al. (2017); } \\
\text { Kuosmanen e } \\
\text { Kortelainen (2005); Mu } \\
\text { et al. (2018); Picazo- } \\
\text { Tadeo et al. (2011) }\end{array}$ & $\begin{array}{c}\text { Utilizada para o cálculo da } \\
\text { variável dependente (índice } \\
\text { de Ecoeficiência) }\end{array}$ \\
\hline CAM & $\begin{array}{l}\text { Média de consumo } \\
\text { ração/KG por suíno }\end{array}$ & $\begin{array}{l}\text { Manual Propriedade } \\
\text { Sustentável (2019) }\end{array}$ & $\begin{array}{c}\text { Utilizada para o cálculo da } \\
\text { variável dependente (índice } \\
\text { de Ecoeficiência) }\end{array}$ \\
\hline CER & $\begin{array}{l}\text { Variável Dummy } \\
0-\quad \text { Não possui } \\
\text { certificação } \\
\text { 1- } \quad \text { Possui } \\
\text { certificação }\end{array}$ & Tran e Goto (2018) & Independente \\
\hline TAM & $\begin{array}{c}\text { Quantidade de hectares } \\
\text { da propriedade }\end{array}$ & $\begin{array}{c}\text { Mu et al. (2018); } \\
\text { Picazo-Tadeo et al. } \\
\text { (2011); van Passel et al. } \\
\text { (2007; 2009). } \\
\text { Grzelak et al. (2019) }\end{array}$ & Controle \\
\hline ESC & $\begin{array}{l}\text { Ensino Fundamental; } \\
\text { Ensino Médio e Ensino } \\
\text { Superior }\end{array}$ & $\begin{array}{l}\text { Picazo-Tadeo et al. } \\
\text { (2011); van } \\
\text { Cauwenbergh et al. } \\
\text { (2007) }\end{array}$ & Controle \\
\hline MDO & $\begin{array}{l}\text { Quantidade de pessoas } \\
\text { que trabalham na } \\
\text { atividade }\end{array}$ & $\begin{array}{l}\text { van Passel et al. (2007; } \\
\text { 2009) }\end{array}$ & Controle \\
\hline REC & $\begin{array}{l}\text { Valor recebido por } \\
\text { carcaça sem os } \\
\text { subsídios }\end{array}$ & $\begin{array}{l}\text { van Passel et al. (2007; } \\
\text { 2009) }\end{array}$ & Controle \\
\hline CAP & $\begin{array}{l}\text { Total do capital da } \\
\text { propriedade }\end{array}$ & $\begin{array}{c}\text { van Passel et al. (2007; } \\
\text { 2009) }\end{array}$ & Controle \\
\hline
\end{tabular}

Legenda: ECO - Índice de ecoeficiência da propriedade EGEE - Emissão de gases de efeito estufa; $\mathrm{CH}$ Consumo hídrico; QSA - Quantidade de suínos alojada; QSV - Quantidade de suínos vendidas; RECV Receita total de vendas (R\$); CAM - Conversão alimentar média; CER - Certificação pelo Programa Propriedade Rural Sustentável; REC - Receita por unidade de carcaça CAP - Capital; TAM - Tamanho (hectares); MDO - Mão-de-obra; ESC - Escolaridade

\section{Cálculo do índice de ecoeficiência e modelo econométrico}


Para o cálculo de ecoeficiência foi utilizado o modelo DEA, um modelo nãoparamétrico desenvolvido por Charnes et al. (1978) que avalia a eficiência relativa das unidades de tomada de decisão (DMUs) integrando indicadores de entradas e saídas. Este modelo é aceito internacionalmente nas pesquisas sobre avaliação da ecoeficiência dos sistemas produtivos agropecuários (MU et al., 2018).

Os índices de ecoeficiência são analisados pela soma das pressões ambientais para identificar se os fatores contribuem para o aumento ou diminuição da ecoeficiência das propriedades. You e Zhang (2016) demonstram que o modelo DEA CCR orientado pelas saídas mede a eficiência total das atividades agropecuárias. O presente estudo utilizou tal modelo para verificar o maior retorno econômico em relação ao consumo dos recursos naturais.

Para a classificação da intensidade dos índices de ecoeficiência foi adotada a métrica de You e Zhang (2016) que categoriza a ecoeficiência em quatro níveis: máxima $(1,00)$, alta $(>0,5)$, moderada $(0,3-0,5)$ e baixa $(<0,3)$. Assim, quanto mais próximo de 1 for o índice de ecoeficiência da propriedade agropecuária, mais eficiente é considerada a propriedade, em relação a utilização dos recursos do capital natural.

Referente ao teste da hipótese H1, elaborou-se o modelo empírico (1):

$$
E_{C C O}=\alpha_{i}+\beta_{1} \text { CERdummy }_{i t}+\beta_{2} \text { TAM }_{i t}+\beta_{3} E S C_{i t}+\beta_{4} M D O_{i t}+\beta_{5} R E C_{i t}+\beta_{6} C A P_{i t}{ }^{+} \varepsilon_{i t}
$$

Em que,

$E C O$ - índice de Ecoeficiência da propriedade $i$ no tempo $t$;

$C E R$ - Certificação da propriedade $i$ no tempo $t$ (variável dummy de certificação pelo Programa Propriedade Rural Sustentável, em que 1 a propriedade possui certificação e 0 caso contrário).

$T A M$ - tamanho da propriedade (hectares) $i$ no tempo $t$;

$E S C$ - nível de escolaridade do gestor da propriedade $i$ no tempo $t$;

$M D O$ - Mão-de-obra da atividade exercida na propriedade $i$ no tempo $t$;

$R E C$ - Receita por unidade de carcaça vendida da propriedade $i$ no tempo $t$;

$C A P$ - Capital total investido na atividade suinícola por propriedade $i$ no tempo $t$.

Foi utilizada a modelagem estatística de dados em painel, com abordagem de Mínimos Quadrados Ordinários precedida pela realização dos testes dos pressupostos do modelo, para a definição do modelo de dados em painel mais adequado. Ainda, foram realizados os testes de Shapiro-Wilk, Breusch-Pagan e vif para verificar a distribuição dos 
resíduos, e a presença de problemas de heterocedasticidade e multicolinearidade, respectivamente.

\section{ANÁLISE DOS RESULTADOS}

\section{Índice de ecoeficiência}

Esta seção apresenta os resultados dos índices de ecoeficiência de cada propriedade obtidos por meio do cálculo DEA CCR, orientado pela saída. No ano de 2015 não há propriedades certificadas, pois, o Programa Propriedade Rural Sustentável foi implementado apenas a partir de 2016 (Tabela 3). Em 2015, todas as propriedades apresentaram índices altos de ecoeficiência (YOU; ZHANG, 2016), já as propriedades consideradas ecoeficientes por meio do cálculo DEA no período de 2015 foram PROP J; PROP F e PROP U , tendo as demais obtido índices abaixo de 1,00..

A PROP C do município de Palmitos tem destaque como ponto negativo com índice de 0,69, o menor obtido no período. Ainda no ano de 2015, detectou-se que apenas o município de Riqueza deixou de apresentar propriedade com índice máximo de ecoeficiência e tendo como maior índice alcançado o da PROP H, 0,96.

Em 2016, o número de propriedades que apresentaram índice máximo de ecoeficiência aumentou. Cabe ressaltar que em 2015, três propriedades apresentaram nível máximo de ecoeficiência, cinco em 2016, onde a maioria possui certificação: PROP D, PROP L e PROP R. Embora a PROP K não possua certificação, apresentou nível máximo de ecoeficiência, e as demais obtiveram níveis altos de ecoeficiência em 2016, acima de 0,5 . 
Tabela 3. Índice de Ecoeficiência

\begin{tabular}{|c|c|c|c|c|c|c|c|c|c|}
\hline & & \multicolumn{2}{|c|}{2015} & \multicolumn{2}{|c|}{2016} & \multicolumn{2}{|c|}{2017} & \multicolumn{2}{|c|}{2018} \\
\hline MUNIC & DMU & CERT & ECO & CERT & ECO & CERT & ECO & CERT & ECO \\
\hline Caibi & PROP J & Não & 1,00 & Sim & 0,89 & Sim & 0,93 & $\mathrm{Sim}$ & 0,98 \\
\hline Caibi & PROP K & Não & 0,90 & Não & 1,00 & Não & 0,91 & Não & 0,89 \\
\hline Caibi & PROP P & Não & 0,84 & Não & 0,87 & Não & 0,87 & Não & 0,91 \\
\hline Caibi & PROP R & Não & 0,87 & Sim & 1,00 & Sim & 0,96 & Sim & 1,00 \\
\hline Palmitos & PROP B & Não & 0,93 & Sim & 0,93 & Sim & 0,98 & Sim & 0,99 \\
\hline Palmitos & PROP C & Não & $0 ., 69$ & Não & 0,81 & Não & 0,92 & Não & 0,90 \\
\hline Palmitos & PROP F & Não & 1,00 & Não & 1,00 & Sim & 1,00 & Sim & 1,00 \\
\hline Palmitos & PROP G & Não & 0,91 & Não & 0,98 & Não & 0,97 & Não & 0,91 \\
\hline Palmitos & PROP I & Não & 0,77 & Não & 0,86 & Não & 0,88 & Não & 0,91 \\
\hline Palmitos & PROP N & Não & 085 & Não & 0,95 & Sim & 1,00 & Sim & 1,00 \\
\hline Palmitos & PROP Q & Não & 0,86 & Sim & 0,91 & Sim & 0,95 & Sim & 0,97 \\
\hline Palmitos & PROP U & Não & 1,00 & Sim & 0,89 & Sim & 0,97 & Sim & 0,95 \\
\hline Palmitos & PROP Y & & & & & & & Não & 0,90 \\
\hline Riqueza & PROP A & Não & 0,81 & Sim & 0,90 & Sim & 0,91 & Sim & 0,93 \\
\hline Riqueza & PROP D & Não & 0,82 & Sim & 1,00 & Sim & 0,98 & Sim & 1,00 \\
\hline Riqueza & PROP H & Não & 0,96 & Sim & 0,91 & Sim & 0,87 & Sim & 1,00 \\
\hline Riqueza & PROP L & Não & 0,87 & Sim & 1,00 & Sim & 1,00 & Sim & 0,88 \\
\hline Riqueza & PROP M & Não & 0,85 & Não & 0,97 & Não & 1,00 & Não & 0,97 \\
\hline Riqueza & PROP O & Não & 0,92 & Não & 0,97 & Não & 0,95 & Não & 0,98 \\
\hline Riqueza & PROP T & Não & 0,80 & Sim & 0,91 & Sim & 0,98 & Sim & 0,99 \\
\hline Riqueza & PROP V & & & & & & & Não & 0,95 \\
\hline Riqueza & PROP W & & & & & & & Não & 0,89 \\
\hline Riqueza & PROP X & & & & & & & Não & 0,95 \\
\hline
\end{tabular}

Todos os municípios apresentaram pelo menos uma propriedade com índice máximo de ecoeficiência, porém os menores valores foram encontrados em Palmitos, PROP C, 0,69. Ressalta-se que a propriedade certificada PROP J, apresentou índice 1,00 em 2015, decrescendo em 2016 para 0,89 (Tabela 3).

Em 2017, as propriedades certificadas que apresentaram nível máximo de ecoeficiência foram: PROP F, PROP L e PROP N, tendo as demais apresentado índices acima de 0,90, considerados níveis altos de ecoeficiência. Destaca-se que a PROP M não possui certificação e índice 1,00 de ecoeficiência e, as PROP P e PROP H, ambas com 0,87, os menores valores encontrados. Observa-se que todas as propriedades obtiveram níveis máximos ou altos de ecoeficiência no período.

Ainda em relação ao período, as propriedades do município de Caibi, não obtiveram níveis máximos de ecoeficiência e, ainda, detém uma das duas propriedades com menor índice. No entanto, destaca-se que Palmitos e Riqueza possuem quatro propriedades com índice 1,00 de ecoeficiência, em sua maioria certificadas. 
Em relação à média das propriedades por municípios pertencentes a amostra, identificou-se que todas apresentaram índices médios acima de 0,90, considerados níveis altos segundo You e Zhang (2016).

No ano de 2018, as propriedades certificadas que demonstraram nível máximo de ecoeficiência foram PROP R; PROP F; PROP N; PROP D; PROP H, em contrapartida, a PROP L apresentou menor índice dentre as certificadas com 0,88 e, as restantes, acima de 0,90. Dentre aquelas não certificadas, apenas a PROP E atingiu nível máximo de ecoeficiência, enquanto as PROP W e PROP K, o menor índice, ambas com 0,89. As demais figuraram acima de $0,90, \operatorname{logo}$, todas as propriedades exibiram nível máximo ou alto de ecoeficiência.

No mesmo período, ao verificar o desempenho das propriedades, destaca-se o município de Palmitos, onde se localizam três daquelas avaliadas com índice 1,00, dentre as quais, duas certificadas e uma não certificada. $\mathrm{Na}$ sequência, Riqueza, com duas propriedades, ambas certificadas, e Caibi, com apenas uma, também certificada pelo Programa Propriedade Rural Sustentável. Constata-se que em 2018, os três municípios obtiveram mesma média anual de 0,95, considerada por You e Zhang (2016) como nível alto de ecoeficiência.

Os resultados apontam que as propriedades rurais cooperativistas suinícolas apresentam níveis altos ou máximos de ecoeficiência (YOU; ZHANG, 2016), ainda que poucas tenham atingido o índice máximo de 1,00, em contraposição aos achados de PicazoTadeo et al. (2011) que encontraram níveis baixos de ecoeficiência ao analisar propriedades leiteiras. Ainda, ressalta-se que os resultados da presente pesquisa discordam dos resultados de Bonfiglio et al. (2017) em que a maioria das propriedades apresentaram índices baixos de ecoeficiência.

Em todos os períodos, a maior parte das propriedades com índices máximos de ecoeficiência é certificada, o que aumenta o retorno econômico-financeiro e corrobora com Tran e Goto (2018) que constataram a melhora nos preços em propriedades certificadas. Os autores afirmam que mesmo com o aumento dos custos de mão-de-obra, os resultados líquidos têm evolução significativa a partir da certificação sustentável.

As propriedades certificadas apresentam níveis máximos ou altos de ecoeficiência, resultados que confirmam o entendimento de Godoy-Durán et al. (2017) ao afirmarem que 
a adoção de programas de certificação e a filiação a associações cooperativistas aprimoram os índices de ecoeficiência.

\section{Análise da influência da certificação no índice de ecoeficiência}

Os resultados da inferência estatística permitem verificar se a certificação pelo Programa Propriedade Rural Sustentável teve maior influência nos índices de ecoeficiência. Na Tabela 5 são apresentados os valores da estatística descritiva dos dados de 2015 a 2018.

Tabela 1. Estatística descritiva

\begin{tabular}{|c|c|c|c|c|c|}
\hline Variável & Obs & Média & Std. Dev. & Min & Max \\
\hline ECO & 80 & 0,93 & 0,0648 & 0,68 & 1,00 \\
\hline TAM & 80 & 22,16 & 10,38 & 6,4 & 45 \\
\hline MDO & 80 & 2,5 & 0,93 & 1 & 4 \\
\hline ESC & 80 & 1,56 & 0,74 & 1 & 3 \\
\hline REC & 80 & 28,77 & 2,95 & 19,42 & 33,41 \\
\hline CAP & 80 & 450.595 & 324,732 & 32,000 & 1.500 .000 \\
\hline
\end{tabular}

Fonte: Dados da Pesquisa (2020)

A análise descritiva aponta que a média do índice de ecoeficiência apresentado pelas propriedades suinícolas cooperativistas para o período de 2015 a 2018 foi de 0,93, considerado alto por You e Zhang (2016) que afirmam que as propriedades que índices totais acima de 0,5 são altos.

As propriedades certificadas pelo Programa Propriedade Rural Sustentável da amostra perfazem $57 \%$ do total. A variável de valor recebido de todas as propriedades analisadas por suíno apresentou uma média de $\mathrm{R} \$ 28,77$ entre 2015 e 2018 e as demais variáveis se mostraram discrepantes ao longo do tempo. O tamanho médio das propriedades é de 22 hectares e a média da mão-de-obra empregada na atividade foi de 2 pessoas por propriedade (em muitas delas familiar) e exercendo mais de uma atividade.

Os testes Shapiro-Wilk e Breusch-Pagan e o vif constatam se os resíduos seguem distribuição normal e são homocedásticos e se há ou não problemas de multicolinearidade. Os resultados encontrados apontaram que os resíduos não seguem a distribuição normal (pvalor $=0,0329)$, apresentam variância constante $(\mathrm{p}$-valor $=0,1399)$ e que não foram encontrados problemas de multicolinearidade (vif médio $=1,14$ ) nas variáveis explicativas. De acordo com Fávero e Belfiore (2017) quando o valor do vif for menor ou igual 4 não há problemas de variância compartilhada ( 
Tabela 2). 
Tabela 2. Testes dos pressupostos da regressão

\begin{tabular}{lcc}
\hline Teste & $\mathbf{p}>\mathbf{f}$ & Pressupostos \\
\hline Shapiro-Wilk & 0,0329 & Normalidade dos resíduos \\
Vif médio & 1,14 & Multicolinearidade \\
Breusch-Pagan & 0,1399 & Homocedasticidade \\
\hline
\end{tabular}

Fonte: Dados da Pesquisa (2020)

$\mathrm{Na}$ sequência, foi estimado o modelo pooled com erros-padrões-robustos para análise das inferências estatísticas. $\mathrm{O} \mathrm{R}^{2}$ de 0,6508 indica que a alteração das variáveis independentes explica 65,08\% da variação do índice de ecoeficiência (Tabela 3).

Tabela 3. Influência da certificação nos índices de Ecoeficiência

\begin{tabular}{lcccccc}
\hline ECO & Coef. & Std. Err. & $\mathbf{t}$ & $\mathbf{p}>|\mathbf{t}|$ & $\mathbf{9 5 \%}$ Conf. & Interval. \\
\hline CER & 0,0227436 & 0,0090353 & 2,52 & 0,014 & 0,0047363 & 0,040751 \\
TAM & 0,0007639 & 0,0004058 & 1,88 & 0,064 & $-0,0000448$ & 0,0015727 \\
MDO & $-0,0018859$ & 0,0055925 & $-0,34$ & 0,737 & $-0,0130317$ & 0,00926 \\
ESC & 0,0015438 & 0,0068563 & 0,23 & 0,822 & $-0,0121207$ & 0,0152084 \\
REC & 0,015179 & 0,0016578 & 9,16 & 0,000 & 0,0118751 & 0,0184829 \\
CAP & $5,35 \mathrm{e}-08$ & $1,70 \mathrm{e}-08$ & 3,15 & 0,002 & $1,97 \mathrm{e}-08$ & $8,74 \mathrm{e}-08$ \\
Cons & 0,4403205 & 0,0484492 & 9,09 & 0,000 & 0,3437614 & 0,5368797 \\
\hline
\end{tabular}

Prob $>\mathrm{F}=0,0000$

$\mathrm{R}^{2}=0,6508$

Legenda: ECO - Índice de Ecoeficiência; CER - Certificação; REC - Receita por unidade de carcaça; TAM Tamanho (Hectares); MOD - Mão-de-obra; ESC - Escolaridade

Fonte: Dados da Pesquisa (2020)

Os resultados não rejeitam H1, (p-valor $=0,014$ ) ao nível de significância de $5 \%$ e corroboram Godoy-Durán et al. (2017) que apontam influência positiva e significativa entre a certificação e associativismo a cooperativas com melhoria nos índices de ecoeficiência e Tran e Goto (2018) que identificam associação positiva da certificação com o aumento dos preços. Picazo-Tadeo et al. (2011) também identificaram a importância das políticas de certificação para a melhoria da sustentabilidade, porém os autores alertam que é necessário analisar a relação custo-benefício para verificar sua viabilidade.

Ao analisar o tamanho das propriedades, notou-se influência positiva ( $\mathrm{p}$-valor $=$ 0,064) nos índices de ecoeficiência ao nível de significância de $10 \%$ e, portanto, quanto maior o tamanho da propriedade, melhor o índice. As evidências se sustentam em Grzelak et al. (2019), Levy e Lubell (2018) e Lubell et al. (2011) que identificam que propriedades maiores possuem melhores índices de sustentabilidade.

A variável receita por unidade de carcaça está associada de maneira positiva e significativa ( $\mathrm{p}$-valor $=0,000)$ com o aumento dos índices de ecoeficiência. Em relação ao nível de escolaridade do gestor das propriedades, não foi detectada influência significativa, o que diverge de Picazo-Tadeo et al. (2011) e van Passel et al. (2007). Também não há 
evidências de influência significativa entre mão-de-obra e índices de ecoeficiência, que corrobora Grzelak et al. (2019) que igualmente não encontrou evidências de que o tipo de mão-de-obra tenha influência sobre os índices.

\section{CONSIDERAÇÕES FINAIS}

O objetivo da pesquisa foi verificar a influência da certificação pelo Programa Propriedade Rural Sustentável nos índices de ecoeficiência das pequenas propriedades suinícolas catarinenses e, para isso se calculou o índice de ecoeficiência pelo método DEA entre 2015 e 2018. Todas as propriedades apresentaram níveis altos ou máximos de ecoeficiência, segundo a métrica de You e Zhang (2016), ou seja, acima de 0,50. A PROP F do munícipio de Palmitos obteve nível máximo de ecoeficiência e pode ser considerada como unidade de benchmark para comparações futuras e, de acordo com Galdeano-Gómez; Aznar-Sánchez; Pérez-Mesa (2013), é necessário porém, observar os critérios de tamanho, localização e atividades desenvolvidas para melhor comparar as unidades de tomada de decisão.

A comparação entre as médias dos índices de ecoeficiência total aponta Caibi como o único munícipio com média acima de 0,90 nos anos analisados. Nos municípios de Palmitos e Riqueza, apenas em 2015 se observou média inferior a 0,90, o que aponta que todos possuem níveis altos, ainda que na média, Caibi tenha apresentado os índices mais equilibrados.

Além disso, ao analisar a estrutura de gestão das propriedades, é possível perceber que o Programa Propriedade Rural Sustentável auxilia os produtores na implementação de controles econômico-financeiros, na melhoria dos procedimentos de manejo e no planejamento de ações futuras. Estas medidas auxiliam os gestores na verificação, sobretudo, da lucratividade de suas propriedades.

Os resultados não rejeitam $\mathrm{H} 1$, logo, as propriedades com os maiores índices de ecoeficiência são aquelas certificadas pelo Programa Propriedade Rural Sustentável corroborando Godoy-Durán et al. (2017), Picazo-Tadeo et al. (2011) e Tran e Goto (2018) que identificam relação significativa da certificação e pertencimento a cooperativas.

Em relação ao tamanho da propriedade, os achados evidenciaram que as de maior porte apresentam índices mais elevados do que as menores, confirmando os resultados de Grzelak et al. (2019), Levy e Lubell (2018) e Lubell et al. (2011) que comprovaram 
associação significativa entre tamanho e índices de ecoeficiência. Além disso, a variável receita por unidade de carcaça apresenta relação positiva e significativa com os índices. Em relação a variável de escolaridade, ao contrário dos achados de Picazo-Tadeo et al. (2011) e van Passel et al.(2007), na presente pesquisa não foi encontrada relação significante entre a escolaridade dos gestores e os índices de ecoeficiência.

As evidências encontradas no estudo demonstram a importância dos programas de certificações para o engajamento e a melhoria das práticas de sustentabilidade na atividade agropecuária. Nota-se que os incentivos presentes na remuneração e o reconhecimento da propriedade, demonstram um aumento de propriedades certificadas de 2015 a 2018. Ainda, em relação ao desempenho de ecoeficiência, conforme o pressuposto do estudo, a certificação demonstrou ter influência na melhoria dos índices de ecoeficiência, devido a processos produtivos mais sustentáveis e a uma maior profissionalização da atividade, por meio da implementação de controles produtivos, financeiros e econômicos exigidos pelo programa.

Como limitação, destaca-se que nem todas as propriedades foram visitadas e que alguns indicadores ambientais não são controlados nas propriedades, como por exemplo, a qualidade do solo. Apesar do estudo ter sido realizado apenas em um Estado brasileiro, os resultados estão em consonância com os estudos internacionais que demonstraram que a ecoeficiência apresenta relevância tanto para os proprietários cooperados, quanto para o meio ambiente e, principalmente, para os consumidores finais. Sugere-se para pesquisas futuras, outros campos de investigação e diferentes atividades agropecuárias (como por exemplo; leite, aves e grãos), além da comparação entre propriedades cooperativistas e não cooperativistas para análise da influência do cooperativismo sobre os índices de sustentabilidade.

\section{REFERÊNCIAS}

BAUM, R.; BIEŃKOWSKI, J. Eco - Efficiency in Measuring the Sustainable Production of Agricultural Crops. p. 1-12, 2020.

BONFIGLIO, A.; ARZENI, A.; BODINI, A. Assessing eco-efficiency of arable farms in rural areas. Agricultural Systems, v. 151, p. 114-125, 2017.

COHEN, D. A próxima revolução verde já está acontecendo. Revista Exame, p. 1-22, 2019.

DANTSIS, T. et al. A methodological approach to assess and compare the sustainability level of agricultural plant production systems. Ecological Indicators, v. 10, n. 2, p. 256- 
263, 2010.

DESIMONE, L. D.; POPOFF, F. Eco-efficiency. [s.l: s.n.].

FÁVERO, L. P.; BELFIORE, P. Manual de Análise de Dados. $1^{\circ}$ ed. Rio de Janeiro: [s.n.].

FIGGE, F.; HAHN, T. Sustainable Value Added - Measuring corporate contributions to sustainability beyond eco-efficiency. Ecological Economics, v. 48, n. 2, p. 173-187, 2004.

FIGGE, F.; HAHN, T. Value drivers of corporate eco-efficiency: Management accounting information for the efficient use of environmental resources. Management Accounting Research, v. 24, n. 4, p. 387-400, 2013.

FREEMAN, A. M.; HAVEMAN, R. H.; KNEESE, A. V. Economics of environmental policy. [s.l.] United States, 1973.

GALDEANO-GÓMEZ, E. et al. Exploring Synergies Among Agricultural Sustainability Dimensions: An Empirical Study on Farming System in Almería (Southeast Spain).

Ecological Economics, v. 140, p. 99-109, 2017.

GALDEANO-GÓMEZ, E.; AZNAR-SÁNCHEZ, J. A.; PÉREZ-MESA, J. C.

Sustainability dimensions related to agricultural-based development: the experience of 50 years of intensive farming in Almería (Spain). International Journal of Agricultural Sustainability, v. 11, n. 2, p. 125-143, 1 maio 2013.

GODOY-DURÁN, Á. et al. Assessing eco-efficiency and the determinants of horticultural family-farming in southeast Spain. Journal of Environmental Management, v. 204, p. 594-604, 2017.

GÓMEZ-LIMÓN, J. A.; SANCHEZ-FERNANDEZ, G. Empirical evaluation of agricultural sustainability using composite indicators. Ecological Economics, v. 69, n. 5, p. 1062-1075, 2010.

GRZELAK, A. et al. Approaching the environmental sustainable value in agriculture : How factor endowments foster the eco-ef fi ciency. Journal of Cleaner Production, v. 241, p. $1-9,2019$.

HUANG, J. et al. Composite eco-efficiency indicators for China based on data envelopment analysis. Ecological Indicators, v. 85, n. April 2017, p. 674-697, 2018.

HUETH, B.; REYNOLDS, A. A Life-Cycle Perspective on Governing Cooperative Enterprises in Agriculture. Choices, v. 26, n. 3, 18 mar. 2011.

HUPPES, G.; ISHIKAWA, M. A framework for quantified eco-efficiency analysis. Journal of Industrial Ecology, v. 9, n. 4, p. 25-41, 2005.

(ISAR), I. S. OF A. AND R. A Manual for the Preparers and Users of Eco-efficiency Indicators. [s.l: s.n.].

JOLLANDS, N.; LERMIT, J.; PATTERSON, M. Aggregate eco-efficiency indices for New Zealand - A principal components analysis. Journal of Environmental Management, v. 73, n. 4, p. 293-305, 2004.

KUOSMANEN, T.; KORTELAINEN, M. Measuring Eco-efficiency of Production with Data. Journal of Industrial Ecology, v. 9, n. 4, p. 59-72, 2005. 
LASSALETTA, L. et al. Future global pig production systems according to the Shared Socioeconomic Pathways. Science of the Total Environment, v. 665, p. 739-751, 2019.

LEVY, M. A.; LUBELL, M. N. Innovation, cooperation, and the structure of three regional sustainable agriculture networks in California. Regional Environmental Change, v. 18, n. 4, p. 1235-1246, 2018.

LEWANDOWSKI, I.; HÄRDTLEIN, M.; KALTSCHMITT, M. Sustainable crop production: Definition and methodological approach for assessing and implementing sustainability. Crop Science, v. 39, n. 1, p. 184-193, 1999.

LOPES, F. Agronegócios Ministério reduz estimativa para o valor da. Disponível em: <https://valor.globo.com/agronegocios/noticia/2019/09/18/ministerio-reduz-estimativapara-o-valor-da-producao-do-campo-para-r-602-bi.ghtml>. Acesso em: 22 set. 2019.

LUBELL, M.; HILLIS, V.; HOFFMAN, M. Innovation, cooperation, and the perceived benefits and costs of sustainable agriculture practices. Ecology and Society, v. 16, n. 4, 2011.

MARCIS, J.; PINHEIRO DE LIMA, E.; GOUVÊA DA COSTA, S. E. Model for assessing sustainability performance of agricultural cooperatives'. Journal of Cleaner Production, v. 234, p. 933-948, 2019.

MU, W. et al. Assessing the impact of uncertainty on benchmarking the eco-efficiency of dairy farming using fuzzy data envelopment analysis. Journal of Cleaner Production, v. 189, p. 709-717, 2018.

NORTON, B. G.; TOMAN, M. A. Sustainability: Ecological and Economic Perspective. Land Economics, v. 73, n. 4, p. 553-568, 1997.

PICAZO-TADEO, A. J.; GÓMEZ-LIMÓN, J. A.; REIG-MARTÍNEZ, E. Assessing farming eco-efficiency: A Data Envelopment Analysis approach. Journal of Environmental Management, v. 92, n. 4, p. 1154-1164, 2011.

RICHTER LONDERO, P.; LOPES STANZANI, L. M.; DOS SANTOS, A. Análise Uma análise da contribuição econômica e social das cooperativas agropecuárias brasileiras pela Demonstração do Valor Adicionado. Revista de Educação e Pesquisa em Contabilidade (REPeC), v. 13, n. 3, p. 291-309, 2019.

SALA, S.; CIUFFO, B.; NIJKAMP, P. A systemic framework for sustainability assessment. Ecological Economics, v. 119, p. 314-325, 2015.

SCHALTEGGER, S.; BURRITT, R. L. Sustainability accounting for companies: Catchphrase or decision support for business leaders? Journal of World Business, v. 45, n. 4, p. 375-384, 2010.

SCHALTEGGER, S.; STURM, A. Ökologische Rationalität: Ansatzpunkte zur Ausgestaltung von ökologieorientierten Managementinstrumenten. Nomos Verlagsgesellschaft mbH, v. 44, n. 4, p. 273-290, 1990.

STERN, D. I. The capital theory approach to sustainability: A critical appraisal. Journal of Economic Issues, v. 31, n. 1, p. 145-173, 1997.

TRAN, D.; GOTO, D. Impacts of sustainability certification on farm income: Evidence from small-scale specialty green tea farmers in Vietnam. Food Policy, n. November, p. 113, 2018. 
VAN CAUWENBERGH, N. et al. SAFE-A hierarchical framework for assessing the sustainability of agricultural systems. Agriculture, Ecosystems and Environment, v. 120, n. 2-4, p. 229-242, 2007.

VAN PASSEL, S. et al. Measuring farm sustainability and explaining differences in sustainable efficiency. Ecological Economics, v. 62, n. 1, p. 149-161, 2007.

VAN PASSEL, S. et al. Sustainable value assessment of farms using frontier efficiency benchmarks. Journal of Environmental Management, v. 90, n. 10, p. 3057-3069, 2009.

VELEVA, V.; ELLENBECKER, M. Indicators of sustainable production: Framework and methodology. [s.l: s.n.]. v. 9

YOU, H.; ZHANG, X. Ecoefficiency of Intensive Agricultural Production and Its Influencing Factors in China: An Application of DEA-Tobit Analysis. Discrete Dynamics in Nature and Society, v. 2016, 2016. 